\title{
Polarization from Be Disks with 1-Armed Density Waves
}

\author{
R. Ignace
}

Physics and Astronomy, University of Glasgow, Scotland, UK

\begin{abstract}
.
The polarization of Be disks that are not axisymmetric is discussed, with results given for one-armed density waves or global disk oscillations (GDO). Polarimetric diagnostics of electron scattering Be disks are presented, including the expected behavior at (a) optical wavelengths where the star is the dominant source of luminosity and (b) infrared wavelengths where the disk is more luminous than the star. Results for detached disks are given. A qualitative discussion of polarization at $\mathrm{H} \alpha$ is also presented.
\end{abstract}

\section{Introduction}

The 90's have seen a steady accumulation of evidence that the disks around $\mathrm{Be}$ stars are non-axisymmetric. The $\mathrm{H} \alpha$ emission lines that form in the disk are known to display $\mathrm{V} / \mathrm{R}$ variations, and in some cases these variations appear periodic (Dachs 1987; Hubert 1994). Many have sought to explain these variations as arising from a 1-armed spiral arm pattern (or a "global disk oscillation" [GDO] as often coined in this conference), including Okazaki (1991, 1996, 1997, these proceedings), Papaloizou, Savonije, \& Henrichs (1992), and Savonije \& Heemskerk (1993). The theory requires that the disk be Keplerian. The 1-armed pattern circuits the disk on typical time scales of years. Support for this model comes from evidence of prograde GDOs (Telting et al. 1994; Mennickent, Sterken, \& Vogt 1997; Telting-these proceedings), $\mathrm{H} \alpha$ line profile shapes (Hummel \& Vrancken 1995; Hummel \& Hanuschik 1997, Hummel-these proceedings), and optical interferometry (Vakili et al. 1998; Berio et al. 1999; Stee-these proceedings). However, there remains one vital technique for confirming and probing the non-axisymmetric Be disks that has not yet been exploited, namely polarization.

\section{Polarization from Non-Axisymmetric Disks}

The GDO model for explaining the observed V/R variations at $\mathrm{H} \alpha$ is a rather special case of non-axisymmetric disks. The 1-armed spiral patterns are point anti-symmetric (e.g., see Fig. 3 of Okazaki 1997). The GDO is just a perturbation of the disk, so that disk material is redistributed or "sloshed" about as the gas undergoes Keplerian motion, hence the total number of scatterers in the disk is conserved. A decrease in density at one point in the disk is mirrored by an equal amount of increase at the point opposite the star. 
The consequence for the polarization is that for any two points equidistant from the star and $180^{\circ}$ apart in azimuth, the combined number of scatterers (i.e., free electrons) is unaltered by the GDO. This is relevant because the position angle of the polarization for these two points is indistinguishable. In an optically thin medium, the polarization scales with the number of scatterers in a volume element, so that in this limit the GDO will produce no change in the polarization! (See Fig. 1.)

However, a variation in polarization owing to the GDO can be expected for several reasons:

1. Stellar occultation could induce polarimetric variability.

2. Be disks are not optically thin, hence multiple scattering must be considered (e.g., see McDavid et al. in these proceedings for Monte Carlo results of pure scattering disks).

3. Be disks are not pure scattering, so that free-free (ff) and bound-free (bf) absorptive opacities must be considered.

4. The Be disks are strong emitters at $\mathrm{H} \alpha$ (and sometimes at other Balmer lines) and show an IR excess. The disk can scatter its own emission ("selfscattering"; see Jones 1979 for early models of this effect) to produce polarization.

The latter point is especially interesting in that for even a pure optically thin scattering disk, a pole-on Be star could show intrinsic polarization variations if scattering of disk emission accounted for. A simplified but instructive model is to think of the star and GDO as effectively a binary system. The dense region acts like a companion source with emissivity proportional to $\rho^{2}$. This companion is somewhat like a "light bulb" embedded in an otherwise axisymmetric disk that orbits around the Be star. The bulb is bright at $\mathrm{H} \alpha$ and the infrared. In fact, at long wavelengths, the "bulb" and disk are the dominant sources of flux. Figure 1 illustrates how intrinsic polarization could be expected if such a system is viewed pole-on. Of course, this binary model approximation is not realistic, and attenuation and occultation effects will turn out to be important in general, but the model does have the advantage that Brown, McLean, \& Emslie (1978) have solved for the polarimetric variability from binaries embedded in optically thin disks. The next section describes the theory to take several of the realistic effects into account and presents results for a case with $\zeta$ Tau type parameters.

\section{The Continuum Polarization}

Using a Stokes vector approach with $I, Q, U$, and $V$, the expression governing the flux of emergent polarized radiation from the star and disk involves a volume integral over the scattering envelope and the star, as given by

$$
\mathcal{F}_{\nu}=\frac{1}{D^{2}} \int_{\text {env }} \mathbf{j}_{\nu}(\mathbf{r}) e^{-\tau_{\mathbf{z}}(\mathbf{r})} d V
$$

where $D$ is distance to the star, $\mathbf{j}_{\nu}$ is the Stokes vector emissivity, $\tau_{\mathrm{z}}$ is the optical depth along a ray to the point at vector position $r$. The geometry is such that the 


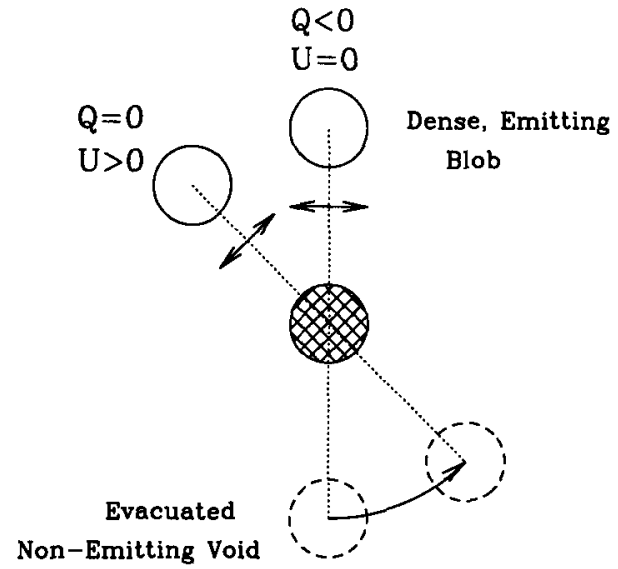

Figure 1. A schematic drawing to illustrate how the polarization from a GDO might act somewhat like a binary. The "blob" (solid) is overdense relative to an axisymmetric disk and emits like $\rho^{2}$. The "hole" (dashed) doesn't emit at all. For pole-on, the degree of polarization is constant, but the polarization position angle should vary with time (with a period half that of the pattern rotation). If the disk is optically thin and the disk emission negligible compared to scattered light, the polarization will not differ from the axisymmetric case, owing to the point anti-symmetry of the GDO.

observer is along the $z$-axis. Cylindrical coordinates with $\left(p_{*}, \varphi_{*}, z_{*}\right)$ for the star and $(p, \phi, z)$ for the observer are used. The disk is generally observed obliquely at inclination $i$ between the $z$ and $z_{*}$ axes, but only pole-on and edge-on cases will be discussed here.

The attenuation through the medium is given by

$$
\tau_{\mathrm{z}}=\int_{z}^{\infty}\left(n_{\mathrm{e}} \sigma_{\mathrm{T}}+\kappa_{\mathrm{ff}} \rho+\kappa_{\mathrm{bf}} \rho\right) d z
$$

for electron number density $n_{\mathrm{e}}$, Thomson scattering cross section $\sigma_{\mathrm{T}}$, and ff and bf opacities $\kappa_{\mathrm{ff}}$ and $\kappa_{\mathrm{bf}}$. The latter opacities are functions of temperature, but an isothermal disk is assumed with $T_{\mathrm{D}}=T_{*}$.

The emissivity can be a rather complicated quantity. It includes direct starlight, direct disk emission, scattered starlight, and scattered disk emission. The methods of Jones (1979) are used to allow for single scattering of disk emission but two scatterings of starlight.

Bjorkman \& Bjorkman (1994) and Wood, Bjorkman, \& Bjorkman (1997) explored respectively models for single scattering (similar to here) and multiple scattering in Be disks with application to WUPPE data for $\zeta$ Tau. The models presented here assume the same overall gross star and disk parameters: $R_{*}=$ $7 R_{\odot}, T_{*}=20,000 \mathrm{~K}$, and constant disk opening angle $\Delta \vartheta_{\mathrm{D}} \approx 3^{\circ}$. Based on IR modeling (Waters 1986), the disk density is taken to decrease with the cube of the radius. For the GDO, a parametrization with $\rho=\rho_{0} g(x, \varphi) x^{-3}$, where the 


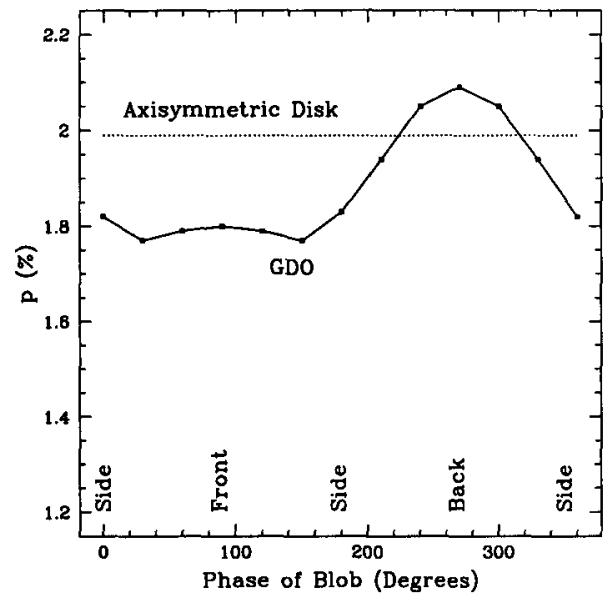

Figure 2. Shown is the variation of percent polarization for an edgeon disk with and without a GDO. The calculation here is for a pure scattering disk (no ff or bf opacities). For the GDO case, the polarization is maximized when the hole is in front of the star (note that bottom indicates the location of the outer dense blob). The peak to trough variation is about $15 \%$.

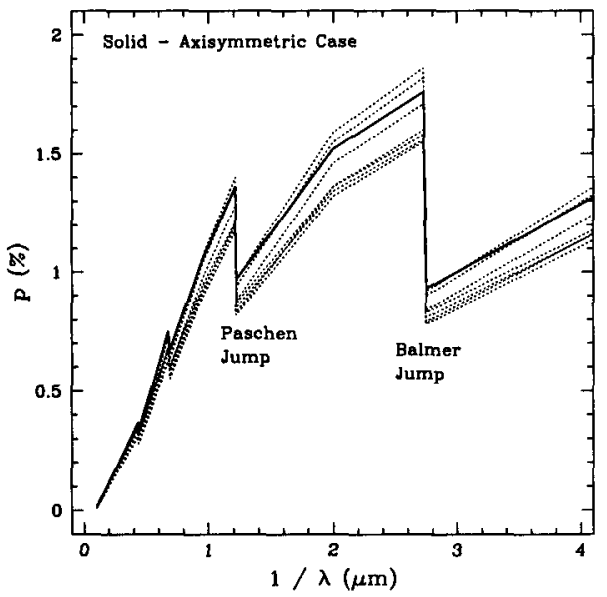

Figure 3. Shown as dotted is wavelength dependent polarization for an edge-on disk at several different phases of the GDO. Solid is for an axisymmetric disk.

constant $\rho_{0}$ is set by the disk optical depth, $x=r / R_{*} \approx p / R_{*}$ for a geometrically thin disk, and $g$ determines the non-axisymmetric structure (see below). Note that the electron, $\mathrm{ff}$, and bf optical depths of the polar wind are negligibly small (for $\zeta$ Tau parameters, $\tau_{\mathrm{e}} \sim 10^{-4}$ and $\tau_{\mathrm{bf}+\mathrm{ff}} \sim 10^{-8}$ at 3 microns) and therefore ignored in the calculations presented here. An optical depth of $\tau_{\mathrm{e}}=4.9$ in the 
equatorial plane of an axisymmetric disk was chosen for a base model that is used in the following calculations. This value of $\tau_{\mathrm{e}}$ roughly matches the Balmer and Paschen jumps observed in the WUPPE data. The combined bf and ff optical depth for this disk is 13.5 at 1 micron.

The $g$ function was selected to roughly approximate the non-axisymmetric perturbation of the circumstellar disk as given in Okazaki (1997) for a nonviscous disk. The chosen form is

$$
g=1+\left(1-0.3 x^{3}\right) e^{1-x} \cos \left(\varphi_{*}-\varphi_{0}\right)
$$

The parameter $\varphi_{0}$ allows for an azimuthal phase shift of the GDO pattern. Note that for arbitrary $\varphi_{*}$, the radial profile of $g$ will show a minimum and a maximum (except for the pathological cases of $\varphi_{*}-\varphi_{0}=\pi / 2$ or $3 \pi / 2$ for which $g=1$ ). There is a density enhancement at small radius and decrement at a few $R_{*}$ for $\varphi_{*}-\varphi_{0}=0$. For $\varphi_{*}-\varphi_{0}=\pi$, it is the opposite case. Values of $g$ range between 0 and 2. The total electron optical depth in the disk is nearly constant with azimuth for this parametrization of $g$.

\subsection{Disks extending down to the star}

Figures 2 and 3 show the expected polarization for an edge-on disk extending down to the star and with $\zeta$ Tau type parameters. The first of these shows a polarization light curve with phase (in degrees) for a pure scattering disk, with the bf and ff opacities set to zero. The points correspond to computed values for a Be disk with a GDO. The dotted line is for an axisymmetric disk. Note that the polarization with a GDO is on average less than for a pure axisymmetric disk. The peak polarization occurs for a geometry where the outer dense region lies opposite the star from the observer. At this phase the attenuation of polarized light (arising primarily from scattering in the plane of the sky) is minimized. The polarization is reduced when the "blob" is on the near side of the star because the attenuation is more severe.

Including the bf and $\mathrm{ff}$ opacities, Figure 3 shows the wavelength dependent polarization as dotted lines with phases between 90 and 270 degrees (at $30^{\circ}$ intervals). Polarization for an axisymmetric disk is shown as solid. The various bound-free edges, such as the Balmer and Paschen jumps, are easily seen. Contrary to expectations, essentially all of the wavelength and phase dependence arises from optical depth effects alone. The contribution of self-scattering to the polarization is extremely small. A calculation for a pole-on viewing perspective of the same disk yields a small polarization, only a few hundreths of a percent.

Self-scattering was much more important in the calculations of Jones (1979), but his assumptions differ significantly from those used in this work. Jones assumed a truncated constant thickness disk of constant density, whereas the disk assumed here is of constant opening angle with decreasing density extending out to infinity. So here, the self-scattering is confined primarily to the inner densest portion of the disk. As a result, (a) the attenuation of polarized light from self-scattering is high and (b) the finite star depolarization effects (Cassinelli, Nordsieck, \& Murison 1987) are severe. Although an exhaustive parameter study was not carried out, a few cases with smaller optical depths were considered (i.e., smaller values of $\rho_{0}$ ), and the contribution by self-scattering was found to be greater, although still only a small fraction of the overall polarization level. 
Table 1. Polarization Values for Detached Disks

\begin{tabular}{cccc}
\hline $\begin{array}{c}R_{\mathrm{D}} \\
\left(R_{*}\right)\end{array}$ & $\begin{array}{c}p \\
\left(\text { phase }=270^{\circ}\right)\end{array}$ & $\begin{array}{c}N_{\mathrm{e}}^{\mathrm{a}}\left(R_{\mathrm{D}}\right) / N_{\text {tot }}^{c} \\
\left.\text { (phase }=270^{\circ}\right)\end{array}$ & $N_{\mathrm{e}}^{b}\left(R_{\mathrm{D}}\right) / N_{\text {tot }}^{c}$ \\
\hline 1.0 & $2.09 \%$ & 1.0 & 1.0 \\
1.25 & $2.21 \%$ & 0.83 & 0.64 \\
1.5 & $2.34 \%$ & 0.67 & 0.44 \\
1.75 & $2.07 \%$ & 0.53 & 0.33 \\
2.0 & $1.80 \%$ & 0.43 & 0.25 \\
2.5 & $1.36 \%$ & 0.28 & 0.16 \\
3.0 & $1.05 \%$ & 0.19 & 0.11 \\
3.5 & $0.81 \%$ & 0.13 & 0.08 \\
5.0 & $0.45 \%$ & 0.050 & 0.040 \\
7.0 & $0.24 \%$ & 0.020 & 0.020 \\
10.0 & $0.13 \%$ & 0.009 & 0.010 \\
\hline \hline
\end{tabular}

${ }^{a}$ Electron column density for a disk with a GDO.

${ }^{b}$ Electron column density for an axisymmetric disk (i.e., $\rho \sim r^{-3}$ ).

${ }^{c} N_{\text {tot }}$ is the electron column density for the case $R_{D}=1$.

\subsection{The case of detached disks}

It has been suggested in this meeting that perhaps magnetic fields are required to regenerate Be disks. Supposing that a magnetic field could enforce co-rotation, a natural expectation is that the inner edge of the disk occurs at the radius where the Keplerian disk speed and the equatorial stellar rotation speed are equal. Hence the disk radius $R_{\mathrm{D}} / R_{*} \approx\left(v_{\text {rot }} / v_{\text {crit }}\right)^{-2}$, where $v_{\text {crit }}$ is the rotational speed of break-up. For typical values of $v_{\text {rot }} / v_{\text {crit }} \sim 0.6-0.7$ for Be stars, the circumstellar disk is detached out to a radius of $2-3 R_{*}$.

The disk polarization for an edge-on viewing perspective was computed for just such detached circumstellar disks. Table 1 summarizes the results for a pure scattering disk (i.e., no bf or ff opacities) with the blob behind the star (phase= $270^{\circ}$; see Fig. 2) as a function of $R_{\mathrm{D}}$. This particular phase was chosen because it corresponds with the phase of maximum polarization for a pure scattering disk extending down to the star. The region intervening the disk and star is assumed a vacuum. Note that the polarization initially increases by evacuating the inner portion, because the inner region of large attenuation is being removed. However, for larger values of $R_{\mathrm{D}}$, the polarization starts to decrease roughly as $R_{\mathrm{D}}^{-2}$, which is expected for optically thin axisymmetric disks.

\section{Considerations of Polarization at $\mathrm{H} \alpha$}

Theoretical results presented so far have been for scattering of continuum flux only, but the polarization across emission line profiles is also of interest. In particular, the $\mathrm{H} \alpha$ emission from the disk can be substantial (otherwise V/R variations wouldn't be observed). The emissivity for line recombination scales as $\rho^{2}$, similar to the bf and ff emissivities responsible for the IR excess. Normally, 
the expectation of strong line emission is to introduce a large component of unpolarized flux leading to a decrease of the polarization across the profile. However, self-scattering of $\mathrm{H} \alpha$ photons may lead to intrinsic line polarization.

There are two major differences between the continum and line profile cases for computing the polarization.

1. Doppler shifts owing to bulk motions of the disk are not a concern for continuum polarization but must be considered for spectral lines. Calculation of the line profile polarization requires that the isovelocity zones (ala Sobolev theory) be determined as seen by each scattering electron.

2. The fact that the line polarization arises from scattering by thermal electrons means that the polarized profile can be severely broadened (e.g., Wood \& Brown 1994; Wood \& Bjorkman 1995).

The differences between processes involved for producing continuum polarization versus those for line profile polarization suggest that self-scattering effects might be more significant in the emission line case than for continuum polarization. Simplistic considerations suggest that the polarization at $\mathrm{H} \alpha$ will still be depressed relative to the continuum polarization, but with some contribution from intrinsic $\mathrm{H} \alpha$ polarization. This is consistent with observations by McLean \& Clarke (1979) and Oudmaijer \& Drew (1999) who find that the polarization across $\mathrm{H} \alpha$ is not sufficiently depressed for some Be and $\mathrm{B}[\mathrm{e}]$ stars, respectively, if the line emission is entirely unpolarized.

\section{Summary}

Results have been presented for the polarization of non-axisymmetric Be disks with GDOs. For the continuum polarization from an edge-on disk, optical depth effects can produce $15 \%$ level variations in the wavelength dependent polarization over the course of half a pattern rotation cycle. The contribution to the polarization from self-scattering is negligible. A pole-on disk is essentially unpolarized. However, multiple scattering effects have been ignored, and the electron scattering optical $\tau_{\mathrm{e}}$ exceeds unity for the case considered. The fact that attenuation effects are large where multiple scattering effects should be important suggests that the results presented here may still be qualitatively correct. Nonetheless, a proper treatment of multiple scattering may reveal that polarization for pole-on disks can be substantial and self-scattering effects more important. Monte Carlo simulations as presented by McDavid et al. (these proceedings) but with the addition of absorptive opacities and associated disk emission will likely be the next future step. It remains to be seen if more detailed calculations reveal that non-axisymmetric pole-on Be disks can be net polarized. If so, stars with low $v \sin i$ values, singly peaked $\mathrm{H} \alpha$ emission lines, large IR excess, and intrinsic polarization may be prime candidates for probing the GDOs of Be disks.

Acknowledgments. The author thanks Jon Bjorkman, Karen Bjorkman, John Brown, Joe Cassinelli, David Clarke, Ken Gayley, and David McDavid for helpful discussions on this topic. This research was supported by a PPARC grant. 


\section{References}

Berio, P., et al. 1999, A\&A, 345203

Bjorkman, J.E., Bjorkman, K.S. 1994, ApJ 436, 818

Brown, J.C., McLean, I.S., Emslie, G.A. 1978

Cassinelli, J.P., Nordsieck, K.H., Murison, M.A. 1987, ApJ 317, 290

Dachs, J. 1987, in Physics of Be Stars, Proc. IAU Coll. 92, (eds.) A. Slettedbak, T.P. Snow (Cambridge University Press: Cambridge), 149

Hubert, A.M. 1994, in IAU Symp. 162, (eds.) L.A. Balona, H.F. Henrichs, J.M. Le Contel (Kluwer: Dordrecht), 341

Hummel, W., Hanuschik, R.W. 1997, A\&A 320, 852

Hummel, W., Vrancken, M. 1995, A\&A 302, 751

Jones, T.J. 1979, ApJ 228, 787

McLean, I.S., Clarke, D. 1979, MNRAS 186, 245

Mennickent, R.E., Sterken, C., Vogt, N. 1997, A\&A 326, 1167

Okazaki, A.T. 1991, PASJ 43, 75

Okazaki, A.T. 1996, PASJ 48, 305

Okazaki, A.T. 1997, A\&A 318, 548

Oudmaijer, R.D., Drew, J.E. 1999, MNRAS 305, 166

Papaloizou, J.C., Savonije, G.J., Henrichs, H.F. 1992, A\&A 265, L45

Savonije, G.J., Heemskerk, M.H.M. 1993, A\&A 276, 409

Telting, J.H., Heemskerk, M.H.M., Henrichs, H.F., Savonije, G.J. 1994, A\&A 288,558

Vakili, F., et al. 1998, A\&A 335, 261

Waters, L.B.F.M. 1986, A\&A 162, 121

Wood, K., Brown, J.C. 1994, A\&A 291, 202

Wood, K., Bjorkman, J.E. 1994, ApJ 443, 348

Wood, K., Bjorkman, K.S., Bjorkman, J.E. 1997, ApJ 477, 926

\section{Discussion}

Ph. Stee: What is the contrast density between the "blobs" and the "holes" that you have used in your simulation?

R. Ignace: The density contrast is extreme for my calculations, in that there exists a point where the perturbation reduces the density to zero, and corresponding increases the density to twice normal at the point opposite the star.

J. Bjorkman: In my simulations of the polarizations produced by $m=1$ (antisymmetric) modes, I also looked at the variations produced by an enhancement on just one side of the star (such as a blob) without the corresponding hole on the opposite side. Such non-antisymmetric density perturbations produce large polarization variations that will be easily observable. I think the fact that the polarization variations are small is strong evidence that the density perturbation associated with $\mathrm{V} / \mathrm{R}$ variations must be antisymmetric like the $m=1$ GDO model. 